\title{
SIMPLY CONNECTED 4-MANIFOLDS NEAR THE BOGOMOLOV-MIYAOKA-YAU LINE
}

\author{
ANDRÁs I. STIPSICZ
}

ABstract. In the following paper, we construct simply connected symplectic 4-manifolds with characteristic numbers satisfying $c_{1}^{2}>8 \frac{9}{10} \chi_{h}$.

\section{Introduction}

The geography problem of compact complex surfaces, (i.e., the characterization of pairs $(a, b) \in \mathbb{Z} \times \mathbb{Z}$ corresponding to minimal complex surfaces via $\chi_{h}=a$ and $c_{1}^{2}=b$,) is a well-studied part of algebraic geometry. (See [Ch1, Ch2, P]. Here $\chi_{h}$ stands for the holomorphic Euler characteristic and $c_{1}^{2}$ for the square of the first Chern class of the surface at hand.) In the light of recent advances in symplectic topology and smooth 4-manifold theory, the same question has been raised for minimal symplectic and for irreducible 4-manifolds. The definitions of the invariants $\chi_{h}$ and $c_{1}^{2}$ has been extended by the formulae

$$
\chi_{h}(X)=\frac{1}{4}(\sigma(X)+e(X)) \text { and } c_{1}^{2}(X)=3 \sigma(X)+2 e(X),
$$

where $X$ is a closed, oriented 4-manifold with odd $b_{1}(X)-b_{2}^{+}(X), \sigma(X)$ is its signature and $e(X)$ is its Euler characteristic. (Note that for a symplectic 4manifold $X$ the difference $b_{1}(X)-b_{2}^{+}(X)$ is always odd.) Results of Gompf and Mrowka [GM], Szabó [Sz], Fintushel and Stern [FS1, FS2], and many others show that the answer for the different classification questions (for complex surfaces, symplectic 4-manifolds, and for smooth irreducible 4-manifolds,) is qualitatively different, even if we assume that - for sake of simplicity - our manifolds are simply connected. Gompf and Mrowka [GM] showed the first examples of simply connected irreducible (in fact, symplectic) 4-manifolds not carrying complex structures, then Szabó $[\mathrm{Sz}]$ found simply connected (irreducible) 4-manifolds with no symplectic structures. These works were followed by constructions of Fintushel and Stern [FS1, FS2] providing hordes of similar examples. (See also [GS, Pa, S1].) All the above examples shared the property that their signature $\sigma$ was negative - equivalently, $c_{1}^{2}<8 \chi_{h}$.

Besides $\mathbb{C P}^{2}$ (with $c_{1}^{2}\left(\mathbb{C P}^{2}\right)=9, \chi_{h}\left(\mathbb{C P}^{2}\right)=1$ and $\sigma\left(\mathbb{C P}^{2}\right)=1$,) complex surfaces of positive signature were hard to find. Using various branched cover constructions, such examples have been constructed in [Ch1, Ch2, H, MT, PPX,

Received January 13, 1998. Revised December 1, 1998.

Supported by the Magyary Zoltán Foundation and OTKA.

Keywords: Symplectic 4-manifolds, geography of 4-manifolds, Lefschetz fibrations 
So]. It is known that if $S$ is a complex surface, then $c_{1}^{2}(S) \leq 9 \chi_{h}(S)$ (the Bogomolov-Miyaoka-Yau inequality); moreover for $S$ differing from $\mathbb{C P}^{2}$, the equality $c_{1}^{2}(S)=9 \chi_{h}(S)$ holds if and only if the unit disk $U=\left\{\left(z_{1}, z_{2}\right) \in\right.$ $\left.\left.\mathbb{C}^{2}|| z_{1}\right|^{2}+\left|z_{2}\right|^{2}<1\right\}$ covers $S$ (implying, in particular, that $c_{1}^{2}(S)=9 \chi_{h}(S)$ for a compact complex surface $S \neq \mathbb{C P}^{2}$ means $\left.\left|\pi_{1}(S)\right|=\infty\right)$. Examples of surfaces with positive signature found by the above authors are either far from the Bogomolov-Miyaoka-Yau (BMY-)line $c_{1}^{2}=9 \chi_{h}$ or have large fundamental groups. In the following we will construct a family $B(d)(d \geq 4)$ of simply connected symplectic 4-manifolds close to the BMY-line.

Theorem 1.1. For $d \geq 4$, the simply connected, symplectic 4-manifolds $B(d)$ given in Section 3 satisfy $c_{1}^{2}(B(d))>8 \frac{9}{10} \chi_{h}(B(d))$ with finitely many exceptions.

Corollary 1.2. There are infinitely many irreducible 4-manifolds (admitting nontrivial Seiberg-Witten invariants,) satisfying $c_{1}^{2}>8 \frac{9}{10} \chi_{h}$.

It is still an open (and intriguing) question whether simply conneceted irreducible (or symplectic) 4-manifolds exist on the BMY-line or behind it, i.e. $X$ with $c_{1}^{2}(X) \geq 9 \chi_{h}(X)$. (Again, we are interested in examples different from $\mathbb{C P}^{2}$.) One has to be careful about asking the violation of the BMY-inequality by an irreducible 4-manifold. By reversing the orientation of, say a minimal elliptic surface we clearly have such an example. Requiring nonvanishing Seiberg-Witten invariants, for example, the question becomes much harder and more interesting.

In Section 2 of the paper we recall the construction of the complex surfaces $H(n)$ lying on the BMY-line; then using $H(n)$ for appropriate $n$ in Section 3 we will construct $B(d)$ and show the properties announced above. We append two standard constructions in Section 4 for sake of completeness.

\section{Surfaces on the BMY-line}

In constructing $H(n)$ we will follow the description given in [Ch2]. Assume that $D$ is a Riemann surface (complex 1 , real 2 dimensional manifold) of genus 2. We take a $\mathbb{Z}_{5}$-action on $D$ generated by $\gamma: D \rightarrow D$ which has exactly 3 fixed points $Q_{1}, Q_{2}$ and $Q_{3}$. (The existence of such an action is shown in the Appendix.) It is easy to see that the quotient of $D$ by this $\mathbb{Z}_{5}$-action is $\mathbb{C P}^{1}$; let us denote the quotient map by $\varphi: D \rightarrow \mathbb{C P}$. The diagonal in $\mathbb{C P}^{1} \times \mathbb{C P}^{1}$ is denoted by $\Delta$; take the inverse image of $\Delta$ in $D \times D$ via $\varphi \times \varphi: D \times D \rightarrow \mathbb{C P}^{1} \times \mathbb{C P}^{1}$ and denote it by $F \subset D \times D$. Note that since $[\Delta]=\left[\mathbb{C P}^{1} \times\{p t\}.\right]+\left[\{p t.\} \times \mathbb{C P}^{1}\right] \in$ $H_{2}\left(\mathbb{C P}^{1} \times \mathbb{C P}^{1} ; \mathbb{Z}\right)$, we have that $[F]=5([D \times\{p t\}]+.[\{p t\} \times D].) \in H_{2}(D \times D ; \mathbb{Z})$.

Lemma 2.1. $F$ consists of the union of 5 complex curves $\left(F_{1}, \ldots, F_{5}\right)$ each diffeomorphic to $D$. Each $F_{i}$ goes through $\left(Q_{1}, Q_{1}\right),\left(Q_{2}, Q_{2}\right)$ and $\left(Q_{3}, Q_{3}\right) \in$ $D \times D$. Moreover, $F_{i}$ intersects $F_{j}$ in $\left(Q_{k}, Q_{k}\right)$ transversally, otherwise these curves are disjoint and $\left[F_{i}\right]^{2}=-2$ for $1 \leq i \leq 5$. 
Proof. The curve $F_{i}$ is the graph of the map $\gamma^{i}: D \rightarrow D$; consequently $\left[F_{i}\right]^{2}=$ $\left[F_{j}\right]^{2}$. Since $F_{5}$ is the diagonal of $D \times D$, we have $\left[F_{5}\right]^{2}=-2$. By the fact that $\left(\sum\left[F_{i}\right]\right)^{2}=(5([D \times\{p t .\}]+[\{p t .\} \times D]))^{2}=50$, the lemma follows.

If we blow up $D \times D$ in the points $\left(Q_{i}, Q_{i}\right)(i=1,2,3)$, the proper transform of $F$ consists of 5 disjoint curves $\tilde{F}_{1}, \ldots, \tilde{F}_{5} \subset D \times D \# 3 \overline{\mathbb{C P}^{2}}$. Since in the second homology group of $D \times D \# 3 \overline{\mathbb{C P}^{2}}$ we have $\left[\tilde{F}_{1}\right]+\ldots+\left[\tilde{F}_{5}\right]=5([D \times\{p t\}]+.[\{p t\} \times$. $D])-5 e_{1}-5 e_{2}-5 e_{3}$ (where $e_{i}$ is the homology class of the exceptional sphere of

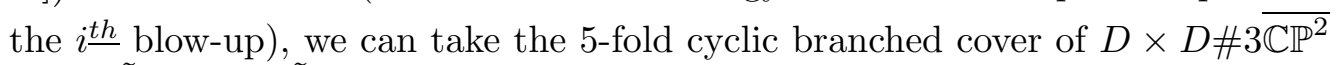
along $\tilde{F}_{1} \cup \ldots \cup \tilde{F}_{5}$. The resulting smooth complex surface is denoted by $H(1)$; the characteristic numbers of $H(1)$ can be easily computed:

Lemma 2.2. The Euler characteristic $e(H(1))$ of $H(1)$ is equal to 75 , its signature is $\sigma(H(1))=25$, hence $c_{1}^{2}(H(1))=225$ and

$$
\chi_{h}(H(1))=\frac{1}{12}\left(c_{2}(H(1))+c_{1}^{2}(H(1))\right)=25 .
$$

Consequently, $c_{1}^{2}(H(1))=9 \chi_{h}(H(1))$, so $H(1)$ is on the Bogomolov-MiyaokaYau line.

Note that the composition of the maps $H(1) \rightarrow D \times D \# 3 \overline{\mathbb{C P}^{2}} \rightarrow D \times D \stackrel{p r}{\longrightarrow} D$ gives a fibration of $H(1)$ over $D$, the regular fiber being a curve which is a 5 -fold cover of $D$ branched in 5 points, hence it is a curve of genus 16. Each $\tilde{F}_{i}$ gives rise to a section of this fibration, the image of this section is a curve of genus 2 with self-intersection -1 .

Any $n$-fold cover of $H(1)$ will be on the BMY-line as well. This can be seen in two ways: The Euler characteristic and the signature are multiplied by $n$ under an $n$-fold cover, so direct computation shows the statement. Alternatively, since $H(1)$ is on the BMY-line (and $H(1) \neq \mathbb{C P}^{2}$ ), the unit disk $U$ is its universal cover, which is the same for any $n$-fold cover, implying that the latter is also on the BMY-line. We define $H(n)$ as a particular $n$-fold cover of $H(1)$ : Take $\phi_{n}: D_{n} \rightarrow D n$-fold cover of $D$ and pull $H(1) \rightarrow D$ back via $\phi_{n}$. The resulting complex surface $H(n)$ obviously fibers over the Riemann surface $D_{n}$ of genus $(n+1)$ with fibers of genus 16 and has the following characteristic numbers:

Proposition 2.3. The Euler characteristic e $(H(n))$ of $H(n)$ is equal to $75 n$, $\sigma(H(n))=25 n$, hence $c_{1}^{2}(H(n))=225 n$ and $\chi_{h}(H(n))=25 n$. The inverse image of a section of $H(1)$ gives a section of $H(n) \rightarrow D_{n}$; the corresponding submanifold is a Riemann surface of genus $(n+1)$ with self-intersection $-n$. (For more about $H(n)$ see [Ch2] or $[\mathrm{GS}]$. ) 


\section{Construction of the 4-manifolds}

Consider a 4-manifold $X$ admitting a Lefschetz fibration $f: X \rightarrow \mathbb{C P}^{1}$ such that the genus of the generic fiber is 16 , moreover $f$ admits a simply connected fiber and a section with self-intersection -1 . An example of such a Lefschetz fibration is given at the end of the Appendix. (For more about Lefschetz fibrations see [GS].) Taking the fiber connected sum of $H(n)$ and $X$ we get a 4-manifold $Y(n)$ still admitting a Lefschetz fibration over $D_{n} . Y(n)$ is symplectic [G1], and has the following characteristic numbers:

Lemma 3.1. Assume that $\sigma(X)=s$ and $e(X)=t$. Then for the 4-manifold $Y(n)$ we have $\sigma(Y(n))=25 n+s, e(Y(n))=75 n+t+60$, consequently, $c_{1}^{2}(Y(n))=225 n+120+3 s+2 t=225 n+120+c_{1}^{2}(X)$, and $\chi_{h}(Y(n))=$ $25 n+15+\frac{1}{4}(s+t)=25 n+15+\chi_{h}(X)$.

By sewing a section of $H(n)$ to a section of $X$ we get a section of $Y(n) \rightarrow D_{n}$; its image $\Sigma_{n}$ turns out to be an embedded surface of genus $(n+1)$ with selfintersection $-(n+1)$. Since for any Lefschetz fibration and preassigned (finite) set of disjoint sections there is a symplectic structure on the 4-manifold making the sections symplectic [G2, GS], we can assume that $\Sigma_{n} \subset Y(n)$ is a symplectic submanifold. Applying the result of the next theorem it will be easy to get a hold on the fundamental group of the 4-manifold $Y(n)$.

Theorem 3.2. If the 4-manifold $M^{4}$ admits a Lefschetz fibration $f: M \rightarrow C$ over the Riemann surface $C$ with connected fibers, with at least one simply connected fiber and with a section $\Sigma$, then the embedding $\Sigma \hookrightarrow M$ induces an isomorphism $\pi_{1}(\Sigma) \cong \pi_{1}(M)$.

Proof. For a Lefschetz fibration there is an exact sequence

$$
\pi_{1}(F) \rightarrow \pi_{1}(M) \rightarrow \pi_{1}(C) \rightarrow \pi_{0}(F),
$$

where $F$ is the generic fiber. Since the fibers are connected, we have $\pi_{0}(F)=0$; by the existence of a simply connected fiber we have that the homomorphism $\pi_{1}(F) \rightarrow \pi_{1}(M)$ is the zero homomorphism. This implies that the projection $M \rightarrow C$ induces an isomorphism $\pi_{1}(M) \rightarrow \pi_{1}(C)$. Since the composition of the section $\tau: C \rightarrow M$ with the above projection as

$$
C \stackrel{\tau}{\longrightarrow} M \stackrel{f}{\longrightarrow} C,
$$

results $i d_{C}$, we get that $\tau$ induces an isomorphism on the fundamental groups, and this proves the lemma.

For $d \geq 4$ let us take $n=\frac{1}{2}(d-1)(d-2)-1 \geq 1$. The smooth holomorphic curve $C_{d} \subset \mathbb{C P}^{2}$ representing $d$-times the generator is a Riemann surface of genus $n+1$; blowing it up $h=d^{2}-n-1=\frac{1}{2}\left(d^{2}+3 d-2\right)$ times we get the curve $\tilde{C}_{d} \subset \mathbb{C P}^{2} \# h \overline{\mathbb{C P}^{2}}$ of genus $n+1$ with self-intersection $n+1$. Forming the symplectic normal connected sum (cf. [G1]) of $\left(Y(n), \Sigma_{n}\right)$ with $\left(\mathbb{C P}^{2} \# h \overline{\mathbb{C P}^{2}}, \tilde{C}_{d}\right)$ 
(recall the definition of $n$ and $h$ in terms of $d$,) we get a symplectic 4-manifold $B(d)$.

Theorem 3.3. The symplectic 4-manifold $B(d)$ is simply connected, and its characteristic numbers are given as follows: $\sigma(B(d))=12 d^{2}-39 d+s+2$, $e(B(d))=40 d^{2}-117 d+62+t$, hence $c_{1}^{2}(B(d))=116 d^{2}-351 d+130+c_{1}^{2}(X)$ and $\chi_{h}(B(d))=13 d^{2}-39 d+16+\chi_{h}(X)$.

Proof. Since the normal circle of $\tilde{C}_{d} \subset \mathbb{C P}^{2} \# h \overline{\mathbb{C P}^{2}}$ is contractible along any of the exceptional spheres, the complement of $\tilde{C}_{d}$ in $\mathbb{C P}^{2} \# h \overline{\mathbb{C P}^{2}}$ is obviously simply connected. Lemma 3.2 shows that each element of $\pi_{1}(Y(n))$ can be represented by a loop contained by the section $\Sigma_{n}$. This, however, implies that the map $\pi_{1}\left(\partial\left(Y(n)-\nu \Sigma_{n}\right)\right) \rightarrow \pi_{1}\left(Y(n)-\nu \Sigma_{n}\right)$ induced by the embedding is a surjection. Now the application of the Seifert-Van Kampen theorem for $B(d)=\left(Y(n)-\nu \Sigma_{n}\right) \cup_{\partial\left(Y(n)-\nu \Sigma_{n}\right)}\left(\mathbb{C P}^{2} \# h \overline{\mathbb{C P}^{2}}-\tilde{C}_{d}\right)$ shows that $B(d)$ is simply connected. The signature of $\mathbb{C P}^{2} \# h \overline{\mathbb{C P}^{2}}$ obviously equals $1-h=2-d^{2}+n=$ $1-d^{2}+\frac{1}{2}(d-1)(d-2)=-\frac{1}{2}\left(d^{2}+3 d-4\right)$, which implies the formula for $\sigma(B(d))$. The Euler characteristic of $\mathbb{C P}^{2} \# h \overline{\mathbb{C P}^{2}}$ equals $3+h=2+d^{2}-n=$ $3+d^{2}-\frac{1}{2}(d-1)(d-2)=\frac{1}{2}\left(d^{2}+3 d+4\right)$, hence $e(B(d))$ can be easily computed; the rest obviously follows.

Proofs of Theorem 1.1 and Corollary 1.2. Now it is easy to see, that

$$
\lim _{d \rightarrow \infty} \frac{c_{1}^{2}(B(d))}{\chi_{h}(B(d))}=\frac{116}{13}>8 \frac{9}{10} .
$$

Consequently $c_{1}^{2}(B(d))>8 \frac{9}{10} \chi_{h}(B(d))$ holds with finitely many exceptions, proving Theorem 1.1. By blowing down $B(d)$ if possible, we end up with a minimal (simply connected) symplectic, hence irreducible 4-manifold $\tilde{B}(d)$. The symplectic structure ensures the nontriviality of the Seiberg-Witten invariants, and since blowing down does not change $\chi_{h}$ and increases $c_{1}^{2}$, the resulting irreducible 4-manifolds $\tilde{B}(d)$ obviously satisfy $c_{1}^{2}(\tilde{B}(d))>8 \frac{9}{10} \chi_{h}(\tilde{B}(d))$ (with finitely many exceptions). This last observation proves Corollary 1.2.

\section{Remarks 3.4.}

- In the notation we did not record the manifold $X$, although $B(d)$ depends on the choice of $X$. Using the 4-manifold provided by the Appendix we get that $c_{1}^{2}(B(d))=116 d^{2}-351 d+70$ and $\chi_{h}(B(d))=13 d^{2}-39 d+17$. Various choices of $X$ give simply connected irreducible 4-manifolds of positive signature with different characteristic numbers. Combining this freedom with the observation described in [S1], one we can prove that most lattice points in $\mathbb{Z} \times \mathbb{Z}$ satisfying $0 \leq b \leq 8 \frac{9}{10}$ a correspond to a simply connected minimal symplectic (hence irreducible) 4-manifold. 
- It can be shown that for $d \geq 4$ the symplectic 4-manifold $B(d)$ is minimal, hence irreducible: Since $Y(n)$ is a relatively minimal Lefschetz fibration over a Riemann surface of positive genus, the result of [S2] applies and shows that $Y(n)$ is minimal. Adapting the method of $W$. Lorek now the minimality of $B(d)$ follows. For sake of brevity we do not give the complete argument here - the a priori necessary blow-downs (resulting in $\tilde{B}(d)$,) provide examples proving our main result.

- The routine exercise of determining the bound $d_{0}$ for which $d \geq d_{0}$ implies $c_{1}^{2}(B(d))>8 \frac{9}{10} \chi_{h}(B(d))$ is left to the reader.

\section{Appendix}

First we will show a $\mathbb{Z}_{5}$-action on $D$ required at the beginning of Section 2 : Take the (singular) curve $A=\left\{\left[x_{0}: x_{1}: x_{2}\right] \in \mathbb{C P}^{2} \mid x_{0}^{5}-x_{1}^{3} x_{2}\left(x_{1}+x_{2}\right)=0\right\}$ in $\mathbb{C P}^{2}$ and blow up $\mathbb{C P}^{2}$ in $[0: 0: 1]$ (the singular point of the curve $A$ ). The proper transform $\tilde{A}$ still has one singular point, but the proper transform $D$ of an additional blow-up will be smooth. Hence we have found a smooth curve $D$ in $\mathbb{C P}^{2} \# 2 \overline{\mathbb{C P}^{2}}$; restricting the blow-down map $\mathbb{C P}^{2} \# 2 \overline{\mathbb{C P}^{2}} \rightarrow \mathbb{C P}^{2}$ to $D$ and composing it with the projection $\mathbb{C P}^{2}-[1: 0: 0] \rightarrow\left\{x_{0}=0\right\} \approx \mathbb{C P}^{1}$ (mapping $\left[x_{0}: x_{1}: x_{2}\right]$ to $\left.\left[0: x_{1}: x_{2}\right]\right)$ we get a map $\varphi: D \rightarrow \mathbb{C P}^{1}$. This map is simply an explicit description of the 5 -fold cyclic branched cover $D \rightarrow \mathbb{C P}^{1}$ branched in three points $Q_{1}, Q_{2}, Q_{3} \in \mathbb{C P}^{1}$. Consequently we have a $\mathbb{Z}_{5}$-action (the generator is denoted by $\gamma: D \rightarrow D$ ) on $D$; the fixed points of $\gamma$ are the inverse images of $Q_{i}(i=1,2,3)$ (still denoted by $Q_{i}$ in $\left.D\right)$. The above $\mathbb{Z}_{5}$-action can be explicitely seen on $A$ as multiplication of $x_{0}$ by a fifth root of unity (providing fixed points $[0: 0: 1],[0: 1: 0]$ and $[0: 1:-1])$. An easy application of the adjunction formula now shows that $D$ has genus 2 .

Next, we show that a Lefschetz fibration with the properties listed at the begining of Section 3 exists. (Recall that we have to find $f: X \rightarrow \mathbb{C P}^{1}$ such that the generic fiber has genus $16, f$ admits a simply connected fiber and it also has a section of square -1.) Let us fix $p_{1}, \ldots, p_{34}, 34$ distinct points in $\mathbb{C P}^{1}$ and take the (singular) curve $G=\bigcup_{i=1}^{2}\left(\mathbb{C P}^{1} \times\left\{p_{i}\right\}\right) \cup \bigcup_{j=1}^{34}\left(\left\{p_{j}\right\} \times \mathbb{C P}^{1}\right) \subset \mathbb{C P}^{1} \times \mathbb{C P}^{1}$. The double branched cover of $\mathbb{C P}^{1} \times \mathbb{C P}^{1}$ branched along $G$ is a singular complex surface, the desingularized of which being diffeomorphic to $\mathbb{C P}^{2} \# 69 \overline{\mathbb{C P}^{2}}$ [GS] Composing the double branched cover map $\rho: \mathbb{C P}^{2} \# 69 \overline{\mathbb{C P}^{2}} \rightarrow \mathbb{C P}^{1} \times \mathbb{C P}^{1}$ with the projection $p r_{2}: \mathbb{C P}^{1} \times \mathbb{C P}^{1} \rightarrow \mathbb{C P}^{1}$ to the second factor, we get a Lefschetz fibration $f: \mathbb{C P}^{2} \# 69 \overline{\mathbb{C P}^{2}} \rightarrow \mathbb{C P}^{1}$. The generic fiber of $f$ is a curve of genus 16 (the double branched cover of $\mathbb{C P}^{1}$ branched in 34 points). There are two singular fibers (originated from the parts $\mathbb{C P}^{1} \times\left\{p_{i}\right\}$ of the branch locus); each singular fiber is a plumbing of 35 spheres along a star-shaped tree, consequently these singular fibers are simply connected. Moreover, the curves $\left\{p_{j}\right\} \times \mathbb{C P}^{1} \subset G$ give rise to 34 sections of $f$, each being a rational curve with self-intersection -1 . 
Consequently $X=\mathbb{C P}^{2} \# 69 \overline{\mathbb{C P}^{2}}$ with the above fibration provides an example of a 4-manifold required in Section 3. Note that for the above $X$ we have $\sigma(X)=-68$ and $e(X)=72$ (hence $c_{1}^{2}(X)=-60$ and $\chi_{h}(X)=1$ ), so the values of $s$ and $t$ and hence the characteristic numbers of the corresponding $B(d)$ are the ones given in Remark 3.4.

Remark 4.1. An alternative way for constructing $X$ with the above properties can be carried out in the following way. Assume that the manifold $N$ is defined by the Kirby diagram consisting of a 0-framed torus knot T $(2,33)$ linked geometrically once with a (-1)-framed unknot. Take the compactified Milnor fiber $M_{c}(2,33,65)$ corresponding to the singularity $x^{2}+y^{33}+z^{65}=0$ and glue it to $N$ along its boundary, so get $X=N \cup_{\partial} M_{c}(2,33,65)$. It can be shown that this closed 4-manifold admits a complex structure and a Lefschetz fibration $f: X \rightarrow \mathbb{C P}^{1}$ with fibers of genus 16 , such that the torus knot $T(2,33)$ gives a (singular) fiber which is obviously simply connected (since it is homeomorphic to $\left.S^{2}\right)$, and the (-1)-framed unlink gives rise to a section of $f$ with self-intersection -1 . Consequently this latter construction provides an alternative choice for $X$. For related constructions of Lefschetz fibrations see [GS].

\section{Acknowledgements}

We would like to thank Zoltán Szabó for helpful discussions.

\section{References}

[Ch1] Z. Chen, On the geography of surfaces - simply connected minimal surfaces with positive index, Math. Ann. 126 (1987), 141-164.

[Ch2] The existence of algebraic surfaces with preassigned Chern numbers, Math. Z. 206 (1991), 241-254.

[FS1] R. Fintushel and R. Stern, Surgery in cusp neighborhoods and the geography of irreducible 4-manifolds, Invent. Math. 117 (1994), 455-523.

[FS2] Knots, links and 4-manifolds, preprint.

[G1] R. Gompf, A new construction of symplectic manifolds, Ann. of Math. 142 (1995), $527-595$.

[G2] , Lecture at MSRI.

[GM] R. Gompf and T. Mrowka, Irreducible 4-manifolds need not be complex, Ann. of Math. 138 (1993), 61-111.

[GS] R. Gompf and A. Stipsicz, An introduction to 4-manifolds and Kirby calculus, book in preparation.

[H] F. Hirzebruch, Chern numbers of algebraic surfaces, Math. Ann. 266 (1984), 351-356.

[MT] B. Moishezon and M. Teicher, Simply connected algebraic surfaces of positive index, Invent. Math. 89 (1987), 601-644.

[Pa] J. Park, PhD thesis, Michigan State University, 1995.

[P] U. Persson, Chern invariants of surfaces of general type, Compositio Math. 43 (1981), $3-58$.

[PPX] U. Persson, C. Peters and G. Xiao, Geography of spin surfaces, Topology 35 (1996), 845-862.

[So] A. Sommese, On the density of ratios of Chern numbers of algebraic surfaces, Math. Ann. 268 (1984), 207-221. 
[S1] A. Stipsicz, A note on the geography of symplectic manifolds, Turkish J. Math. 20 (1996), 135-139.

[S2] _ Chern numbers of certain Lefschetz fibrations, to appear in the Proceedings of the AMS.

[Sz] Z. Szabó, Simply connected irreducible 4-manifolds with no symplectic structures, to appear in Invent. Math.

Department of Analysis, Elte TTK, 1088. Múzeum Krt. 6-8., Budapest, Hungary E-mail address: stipsicz@cs.elte.hu 